\title{
Miniinvasive interventional bridge to major surgical repair of critical aortic coarctation in a newborn with severe multiorgan failure
}

\author{
Ireneusz Haponiuk ${ }^{1}$, Maciej Chojnicki ${ }^{1}$, Mariusz Steffens ${ }^{1}$, Radosław Jaworski ${ }^{1}$, Aneta Szofer-Sendrowska ${ }^{1}$, Jacek Juscinski ${ }^{1}$, \\ Ewelina Kwasniak'1, Konrad Paczkowski', Jacek Zielinski², Katarzyna Gierat-Haponiuk ${ }^{3}$ \\ 1Department of Paediatric Cardiac Surgery, Mikolaj Kopernik Pomeranian Centre of Traumatology, Gdansk, Poland \\ 2Department of Oncological and Plastic Surgery, Medical University of Gdansk, Poland \\ 32Department of Rehabilitation, Medical University of Gdansk, Poland
}

Videosurgery Miniinv 2013; 8 (3): 244-248

DOI: $10.5114 /$ wiitm.2011.33472

\begin{abstract}
We present a case of a severely ill newborn with complex coarctation, multiorgan failure and massive oedema, who was treated with emergency stenting of the isthmus on the second day of life, which was followed by surgical stent removal and repair of the arch on the 29th day, after stabilization of his general status. Interventional percutaneous direct stent implantation was performed, using a coronary stent (Abbott Multi-Link Vision Coronary Stent $3.5 \mathrm{~mm} /$ $15 \mathrm{~mm}$, USA) to cover the area of the aortic isthmus in the newborn. The area from the origin of the left subclavian artery to the beginning of the descending thoracic aorta beneath the isthmus was widely expanded. Control angiography showed normal size of the isthmus without a systolic gradient in the area. In the next 3 weeks the boy improved his general status, with normalization of liver and renal parameters, as well as resolution of the oedema, and underwent surgery on his 29th day of life. The procedure of stent removal with aortic extended end-to-end anastomosis was performed without complications, and the infant was transferred to general paediatrics for further treatment. The strategy of miniinvasive interventional bridge to postpone major surgical repair was effective in the presented infant, with positive final results of both cardiological intervention and subsequent surgical repair.
\end{abstract}

Key words: aortic coarctation, paediatric cardiac surgery, interventional cardiology, stent implantation, newborns.

\section{Introduction}

Aortic coarctation (COA) constitutes the fifth most common congenital heart defect with the incidence of 1 in 2500 live births, which affects more male than female infants [1]. Critical coarctation in newborns usually presents with a narrowing of the aortic isthmus, which adversely effects the heart function and decreases peripheral perfusion. Dynamic deterioration of the patient usually coexists with spontaneous closure of the patent arterial duct (PDA), which could be open after intravenous prostaglandin administration, with temporary improvement of the blood flow, and consequently the condition of the infant [2]. The treatment of choice is surgical resection of narrowed segments of the isthmus completed with several types of aortic arch anastomosis, recently with the most reasonable 'extended end-to-end' technique.

The complexity of problems with critically ill newborns with COA that deteriorate despite maximal 
intensive management usually forces the medical staff to continue pharmacological therapy and postpones, or even renders impossible, any surgical intervention. The strategy of prolonged pharmacological treatment, although temporarily effective, does not resolve the anatomical cause of the disease.

We present a case of a severely ill newborn with complex coarctation, multiorgan failure and massive oedema, who was treated with emergency stenting of the isthmus on the second day of life, which was followed by surgical stent removal and repair of the arch on the $29^{\text {th }}$ day, after stabilization of his general status. We followed the strategy of minimally invasive interventional bridging of a borderline patient with regard to the experience of stenting of native coarctations in older patients, or complex recurrent stenosis of previously surgically treated children [3].

\section{Case report}

The critically ill male newborn was referred for surgical treatment of aortic coarctation on his second day of life (body weight $3.5 \mathrm{~kg}$ ). The boy suffered from general oedema of undefined cause. He was initially treated with bilateral pleural punctures in the neonatology department. Prostaglandin $E_{1}$ intrave- nous infusion, antibiotics and steroids were administered. The patient was referred for emergency surgery. Despite maximal pharmacological treatment the symptoms of arterial duct constriction with tension hydrothorax and clotting disturbances with diffuse bleeding sites caused the need to postpone the surgery because of independent contraindications. Subsequently drainage of both pleural cavities was performed; the evacuation of $1000 \mathrm{ml}$ of effusion fluid was noted during the first $24 \mathrm{~h}$. The interventional procedure with intra-aortic stent implantation was a reasonable miniinvasive alternative treatment to reach temporary stabilization of aortic flow, and postpone the major surgery until the general status of the child is improved. Interventional percutaneous direct stent implantation was performed, using a coronary stent (Abbott Multi-Link Vision Coronary Stent $3.5 \mathrm{~mm} / 15 \mathrm{~mm}$, USA) to cover the area of the aortic isthmus from the origin of the left subclavian artery to the beginning of the descending thoracic aorta beneath the isthmus (Photo 1). After the procedure prostaglandin $\mathrm{E}_{1}$ infusion was stopped, and lowdose intravenous heparin was administered.

Control echocardiography proved the good effect of the aortic intervention, with left ventricular func-
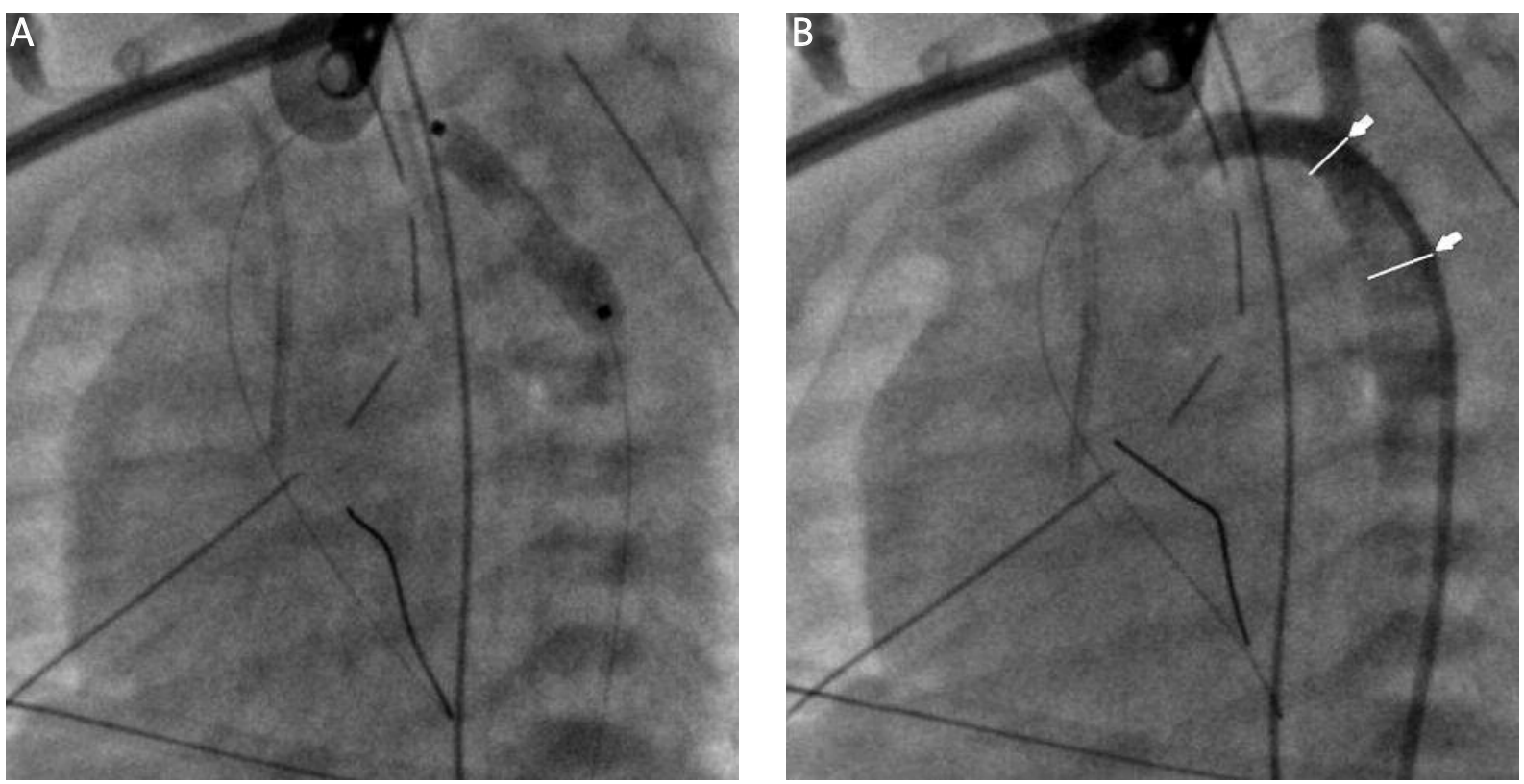

Photo 1. Percutaneous coronary stent implantation (Abbott Multi-Link Vision Coronary Stent $3.5 \mathrm{~mm} / 15 \mathrm{~mm}$, USA): A - Stent implantation. B - Aortography after intervention; note the coronary stent is covering the area of the aortic isthmus from the origin of the left subclavian artery to the beginning of the descending thoracic aorta beneath the isthmus (arrows) 

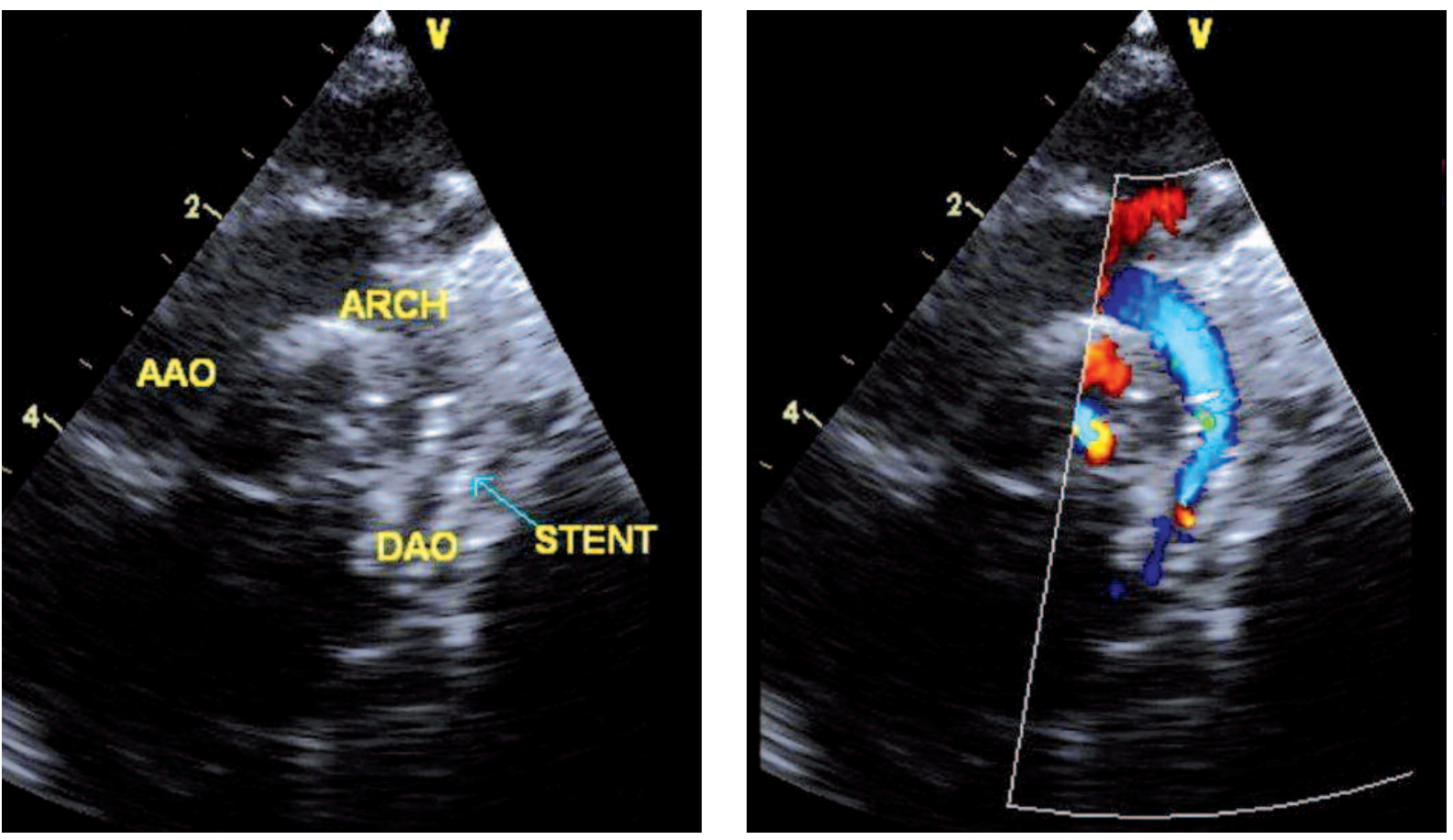

Photo 2. Echocardiographic image showing right placed stent (arrow) in aortic isthmus without any gradient flow $A A O$ - ascending aorta, $A R C H$ - aortic arch, DAO - descending aorta

tion recovery, and ductus arteriosus (PDA) closure in the area of the implanted stent (Photo 2).

In the next 3 weeks the boy improved his general status, with normalization of liver and renal parameters, as well as resolution of the oedema. He was referred for surgery on his $29^{\text {th }}$ day of life; the procedure of stent removal with aortic extended end-to-end anastomosis was performed. Routine non-invasive and invasive monitoring were applied. Prophylactic antibiotic was administered a $0.5 \mathrm{~h}$ prior to general anaesthesia. Induction of general anaesthesia (GA) was achieved by both inhalation of volatile anaesthetic and intravenous administration of a hypnotic drug, while maintenance of GA was achieved by volatile anaesthetic and continuous administration of analgesic drugs and neuromuscular blocking drugs. The patient was extubated $4 \mathrm{~h}$ after the procedure without any complications, and sent to general paediatrics on postoperative day 4. Control echocardiography showed a good final effect of aortic surgery (Photo 3).

\section{Discussion}

The treatment of choice for critical duct-dependent aortic coarctation (CoA) is anatomical repair in a newborn. Surgical resection of narrowed segments with primary anastomosis created to broaden the isthmic area as well as the distal aortic arch has become the gold standard in critically ill infants, initially treated with prostaglandins [4]. Questions arise when the child presents with severe heart failure and generally poor condition, suboptimal body weight, congenital infection or other endocrinological problems [5]. The complexity of accompanying problems sometimes overcomes the coarctation itself, and prevents any surgery, necessitating postponement of the operation, to avoid additional operative trauma accompanying the repair in such settings [1]. However, primary stent implantation for palliation of coarctation of the aorta is technically feasible in preterm neonates and young infants, in whom surgery may be either risky, or relatively contraindicated for a variety of reasons $[6,7]$.

Interventional procedures were successfully adopted to cooperate with surgery in a common hybrid strategy for the treatment of congenital intracardiac and cardiovascular defects [8-10]. Balloon angioplasty for CoA became popular in older children with the advent of non-surgical interventional approaches to congenital cardiac defects, with good initial results 

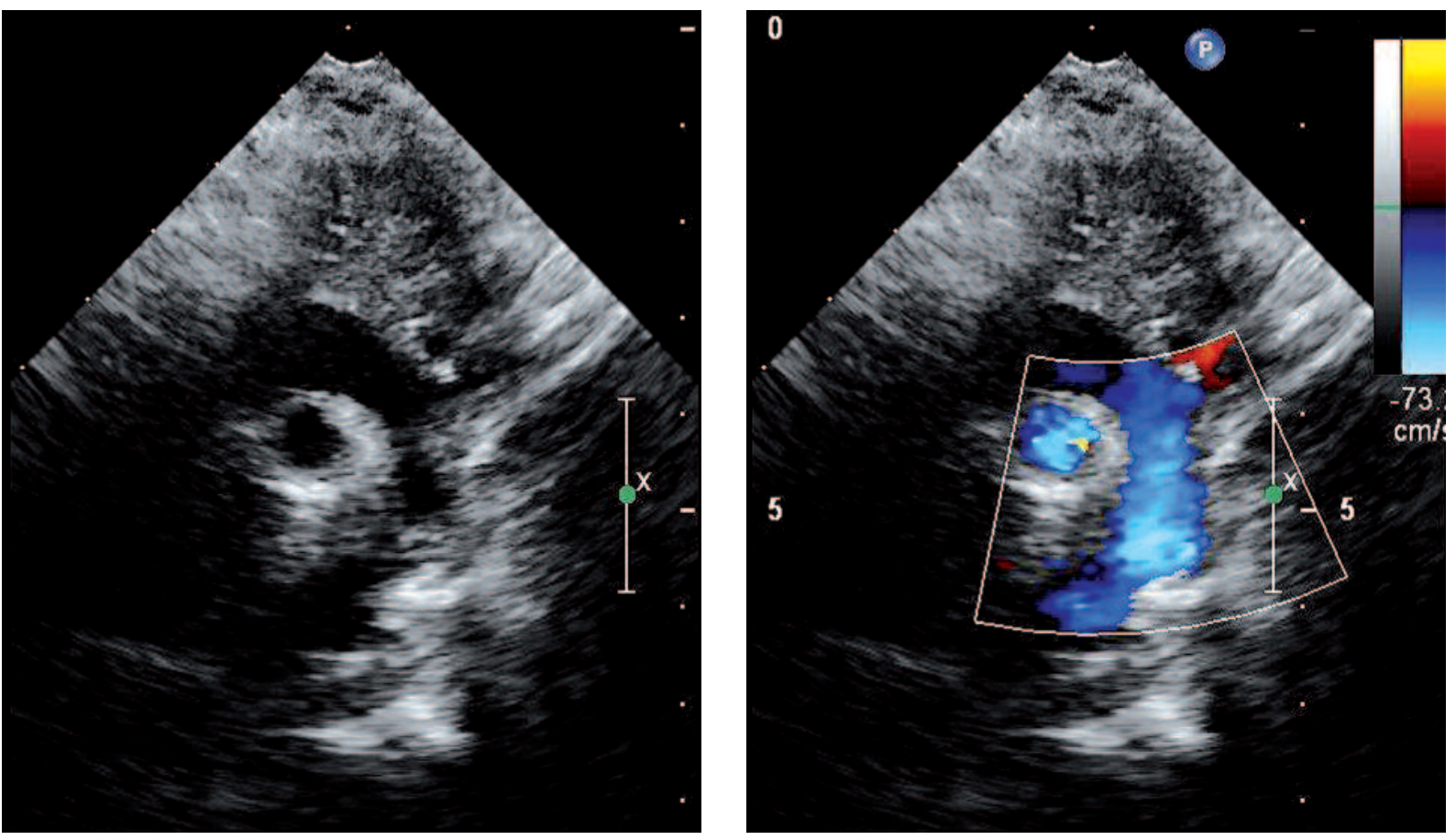

Photo 3. Postoperative echocardiography proved normal aortic blood flow without any gradient in the aortic isthmus

and high rates of restenosis, as well as aneurysm formation [1]. Stent technology has evolved recently. These techniques include primarily balloon expanding and stent implantation, with good short-term results and still poor long-term evaluation [11].

In the present case there was no option of early surgery because the expected potential advantages were less than the predictable risk of early critical complications related to severe multiorgan failure and additional surgical trauma [12]. Therefore the first procedure was emergency bilateral thoracic drainage to evacuate the fluid produced in unknown mechanisms from the birth. Despite the drainage the infant was still in severe insufficiency, with the need to prolong mechanical ventilation and inotropic support, with concomitant symptoms of clotting system disturbances, and liver and renal failure. The decision of miniinvasive interventional bridge to major surgical repair of critical aortic coarctation was undertaken in a multidisciplinary team in the settings of rapid deterioration of the infant and uncontrolled severe heart failure symptoms. The procedure of fluoroscopy-controlled stent implantation was performed without complications, and the prostaglandin $E_{1}$ was stopped. In the next 3 weeks the general condition of the boy improved, the child was extubated after compensa- tion of multiorgan function, and the inotropic support was reduced to a regular range. The child was referred for surgical removal of the stent with coarctation repair, which was successfully performed on the $29^{\text {th }}$ day of life.

The strategy of miniinvasive interventional bridge to postpone major surgical repair proved effective in the presented infant. The positive final results of both cardiological intervention and subsequent surgical repair are promising, and encourage continuation of the strategy in subsequent borderline patients.

\section{References}

1. Kenny D, Hijazi ZM. Coarctation of the aorta: from fetal life to adulthood. Cardiology J 2011; 16: 487-95.

2. Allan LD, Chita S, Anderson RH, et al. Coarctation of the aorta: an echocardiographic, anatomical, and functional study. Br Heart J 1987; 57: 65-6.

3. Holzer RJ, Chisolm JL, Hill SL, Cheatham JP. Stenting complex aortic arch obstructions. Cathet Cardiovasc Interv 2008; 71: 375-82.

4. Luijendijk P, Bouma BJ, Groenink M, et al. Surgical versus percutaneous treatment of aortic coarctation: new standards in an era of transcatheter repair. Expert Rev Cardiovasc Ther 2012; 10: 1517-31.

5. Kazmierczak P, Moll JA, Moll JJ. Surgical treatment of coarctation of the aorta in a neonate with body weight of 1200 g. Kardiochirur Torakochi 2010; 7: 27-9. 
6. Sreeram I, Sreeram N, Bennink G. Palliative stent implantation for coarctation in neonates and young infants. Ann Pediatr Card 2012; 5: 145-50.

7. Cools B, Meyns B, Gewillig M. Hybrid stenting of aortic coarctation in very low birth weight premature infant. Catheter Cardiovasc Interv 2013, 81: E 195-8.

8. Haponiuk I, Chojnicki M, Jaworski R, et al. Miniinvasive hybrid closure of multiple muscular ventricular septal defects in premature infant with a novel use of Amplatzer Duct Occluder II a case report. Videosurgery Miniinv 2011; 6: 33-6.

9. Haponiuk I, Chojnicki M, Jaworski R, et al. Delayed closure of multiple muscular ventricular septal defects in an infant after coarctation repair and a hybrid procedure: a case report. Heart Surg Forum 2011; 14: E67-9.

10. Chojnicki M, Haponiuk I, Jaworski R. Intraoperative imaging of hybrid procedure for muscular ventricular septal defects closure with Amplatzer Duct Occluder II. Kardiol Pol 2011; 69: 1280-1.

11. Kenny D, Cao QL, Kavinsky CJ, Hijazi ZM. Innovative resource utilization to fashion individualized covered stents in the setting of aortic coarctation. Cathet Cardiovasc Intervent 2011; 78: 413-8.

12. Haponiuk I, Chojnicki M, Jaworski R, et al. Miniinvasive hybrid procedure for device migration after percutaneous closure of persistent arterial duct: a case report. Videosurgery Miniinv 2012; 7: 202-5.

Received: 5.10.2012, accepted: 2.02.2013. 